\title{
Computing GCRDs of Approximate Differential Polynomials
}

\author{
Mark Giesbrecht and Joseph Haraldson \\ Cheriton School of Computer Science \\ University of Waterloo \\ Waterloo, Ontario \\ \{mwg,jharalds\}@uwaterloo.ca
}

June 17, 2021

\begin{abstract}
Differential (Ore) type polynomials with approximate polynomial coefficients are introduced. These provide a useful representation of approximate differential operators with a strong algebraic structure, which has been used successfully in the exact, symbolic, setting. We then present an algorithm for the approximate Greatest Common Right Divisor (GCRD) of two approximate differential polynomials, which intuitively is the differential operator whose solutions are those common to the two inputs operators. More formally, given approximate differential polynomials $f$ and $g$, we show how to find "nearby" polynomials $\widetilde{f}$ and $\widetilde{g}$ which have a non-trivial GCRD. Here "nearby" is under a suitably defined norm. The algorithm is a generalization of the SVD-based method of Corless et al. (1995) for the approximate GCD of regular polynomials. We work on an appropriately "linearized" differential Sylvester matrix, to which we apply a block SVD. The algorithm has been implemented in Maple and a demonstration of its robustness is presented.
\end{abstract}




\section{Introduction}

The ring of differential (Ore) polynomials $\mathbb{R}(t)\left[\partial ;^{\prime}\right]$ over the real numbers $\mathbb{R}$ provides a (non-commutative) polynomial ring structure to the linear ordinary differential operators. Differential polynomials have found great utility in symbolic computation, as they allow us to apply algebraic tools to the simplification and solution of linear differential equations; see Bronstein and Petkovšek (1994) for a nice introduction to the mathematical and computational aspects. The ring of differential polynomials $\mathbb{R}(t)\left[\partial ;^{\prime}\right]$ is defined as the usual polynomials in $\mathbb{R}(t)[\partial]$ (i.e., polynomials in $\partial$ over the commutative field of rational functions $\mathbb{R}(t))$, under the usual polynomial addition and the non-commutative multiplication rule

$$
\partial f(t)=f(t) \partial+f^{\prime}(t) \text { for } f(t) \in \mathbb{R}(t),
$$

where $f^{\prime}(t)$ is the usual formal derivative of $f(t)$ with respect to $t$. This definition of $\mathbb{R}(t)\left[\partial ;^{\prime}\right]$ is useful because there is a natural action of $\mathbb{R}(t)\left[\partial ;^{\prime}\right]$ on the space $\mathcal{C}^{\infty}$ of infinitely differentiable functions $y(t): \mathbb{R} \rightarrow \mathbb{R}$. In particular, for any $y(t) \in \mathcal{C}^{\infty}$,

$$
f(\partial)=\sum_{0 \leq i \leq k} f_{i}(t) \partial^{i} \text { acts on } y(t) \text { as } \sum_{0 \leq i \leq k} f_{i}(t) \frac{d^{i}}{d t^{i}} y(t) .
$$

A primary benefit of viewing differential operators in this way is that they have the structure of a left (and right) Euclidean domain. In particular, for any two polynomials $f, g \in \mathbb{R}(t)\left[\partial ;^{\prime}\right]$, there is a unique polynomial $h \in$ $\mathbb{R}(t)\left[\partial ;^{\prime}\right]$ of maximal degree in $\partial$ such that $f=u h$ and $g=v h$ for $u, v \in$ $\mathbb{R}(t)\left[\partial ;^{\prime}\right]$ (i.e., $h$ divides $f$ and $g$ exactly on the right). This polynomial $h$ is called the GCRD of $f$ and $g$ and it is unique up to a scalar multiplication by a non-zero element of $\mathbb{R}(t)$ (we could make this GCRD have leading coefficient 1 , but that would introduce denominators from $\mathbb{R}[t]$, as well as potential numerical instability, as we shall see).

The important geometric interpretation is that there is an algorithm to determine the differential polynomial whose solution spaces is the intersection of the solution space of $f$ and $g$; this is precisely $h=\operatorname{gcrd}(f, g)$.

The goal of this paper is to devise an efficient, numerically robust algorithm to compute the GCRD when the coefficients in $\mathbb{R}$ are given approximately. Specifically, given $f, g \in \mathbb{R}(t)\left[\partial ;^{\prime}\right]$, we wish to find $\widetilde{f}, \widetilde{g} \in \mathbb{R}(t)\left[\partial ;^{\prime}\right]$, where $\widetilde{f}$ is "near" $f$ and $\widetilde{g}$ is "near" $g$ such that $\operatorname{deg}_{\partial} \operatorname{gcrd}(\widetilde{f}, \widetilde{g}) \geq 1$. That 
is, $\tilde{f}$ and $\widetilde{g}$ have a non-trivial GCRD. The precise definition of nearness is given below. The approach we will take is similar to the one developed in Corless et al. (1995) for regular polynomials, which is to reduce the problem to a singular value decomposition or total least squares problem.

The problem of computing the GCRD in a symbolic and exact setting dates back to Ore (1933), who presents a Euclidean like algorithm. See Bronstein and Petkovšek (1994) for an elaboration of this approach. Li and Nemes (1997) introduce a differential-resultant-based algorithm which makes computation of the GCRD very efficient using modular arithmetic. We generalize and adapt their approach to a numerical setting here.

The analogous approximate GCD problem for approximate regular (commutative) polynomials has been a key topic of research in symbolic-numeric computing since its inception. A full survey is not possible here, but we note the deep connection between our current work and that of Corless et al. (1995); see also Karmarkar and Lakshman (1996), Sasaki and Sasaki (1997), and Zeng and Dayton (2004). Also important to this current work is the use of so-called structured (numerical) matrix methods for approximate GCD, such as structured total least squares (STLS) and structured total least norm (STLN); see Botting et al. (2005) and Kaltofen et al. (2005). A structured approach to relative primality is taken in Beckermann and Labahn (1998). More directly employed later in this paper is the multiple polynomial approximate GCD method of Kaltofen et al. (2006). This latter paper also provides a nice survey of recent developments.

\subsection{Differential polynomial basics}

The ring $\mathbb{R}(t)\left[\partial ;^{\prime}\right]$ is a non-commutative principal left (and right) ideal domain. For $f, g \in R(t)\left[\partial ; ;^{\prime}\right]$, with $\operatorname{deg}_{\partial} f=n$ and $\operatorname{deg}_{\partial} g=m$, we have the following properties (Ore, 1933).

1. $\operatorname{deg}_{\partial}(f g)=\operatorname{deg}_{\partial} f+\operatorname{deg}_{\partial} g$, $\operatorname{deg}_{\partial}(f+g) \leq \max \left\{\operatorname{deg}_{\partial} f, \operatorname{deg}_{\partial} g\right\}$.

2. There exist $q, r \in \mathbb{R}(t)\left[\partial ;^{\prime}\right]$ with $\operatorname{deg}_{\partial} r<\operatorname{deg}_{\partial} g$ such that $f=q g+r$ (Division with Remainder).

3. There exists $h \in \mathbb{R}(t)\left[\partial,^{\prime}\right]$ of maximal degree in $\partial$ with $f=w_{1} h$ and $g=w_{2} h . h$ is called the GCRD (Greatest Common Right Divisor) of $f$ and $g$. 
4. There exist $w_{3}, w_{4} \in R(t)\left[\partial,^{\prime}\right]$ such that $w_{3} f=w_{4} g=h$ for $h$ of minimal degree. $h$ is called the LCLM (Least Common Left Multiple) of $f$ and $g$.

5. $\operatorname{deg}_{\partial} \operatorname{lclm}(f, g)=\operatorname{deg}_{\partial} f+\operatorname{deg}_{\partial} g-\operatorname{deg}_{\partial} \operatorname{gcrd}(f, g)$.

These immediately imply the following characterization of a non-trivial GCRD.

Lemma 1.1. Suppose $f, g \in \mathbb{R}(t)\left[\partial ;^{\prime}\right]$, with $\operatorname{deg}_{\partial} f=m$ and $\operatorname{deg}_{\partial} g=n$. Then $\operatorname{deg}_{\partial} \operatorname{gcrd}(f, g) \geq 1$ if and only if there exists $u, v \in \mathbb{R}(t)\left[\partial ;^{\prime}\right]$ such that $\operatorname{deg}_{\partial} u<n, \operatorname{deg}_{\partial} v<m$, and $u f+v g=0$.

This lemma will enable us to set up a resultant-like linear system over $\mathbb{R}$ for the GCRD, which will lead to the desired algorithms.

Because of the non-commutative property of $\mathbb{R}(t)\left[\partial ;^{\prime}\right]$, it will be important to maintain a canonical form for any $f \in \mathbb{R}(t)\left[\partial ;^{\prime}\right]$. We will always write

$$
f=\frac{1}{f_{-1}(t)} \sum_{i=0}^{m} f_{i}(t) \partial^{i},
$$

for polynomials $f_{-1}, f_{0}, \ldots, f_{m} \in \mathbb{R}[t]$, with coefficients in $\mathbb{R}(t)$ always written to the left of powers of $\partial$. Moreover, for $f$ as above, and $\ell>\operatorname{deg} f$, we define

$$
\Psi_{\ell}(f)=\frac{1}{f_{-1}} \cdot\left(f_{0}, f_{1}, \ldots, f_{m}, 0, \ldots, 0\right) \in \mathbb{R}(t)^{\ell} .
$$

I.e., $\Psi_{\ell}$ maps polynomials in $\mathbb{R}(t)\left[\partial ;^{\prime}\right]$ of degree (in $\partial$ ) less than $\ell$ into $\mathbb{R}(t)^{\ell}$. It will also be useful to ensure that our differential polynomials are primitive.

Definition 1.2 (Primitive Differential Polynomial). Let $f \in \mathbb{R}[t]\left[\partial ;^{\prime}\right]$ where $\operatorname{deg}_{\partial} f=m$ is in standard form. The content of $f$ is given by $\operatorname{cont}(f)=$ $\operatorname{gcd}\left(f_{0}, f_{1}, \ldots, f_{m}\right)$. If cont $(f)=1$, we say that $f$ is primitive.

For our primary problem of computing GCRDs of differential polynomials $f, g \in \mathbb{R}(t)\left[\partial ;^{\prime}\right]$, we will assume both that the coefficients of $f, g$ are polynomials in $\mathbb{R}[t]$, and that $f$ and $g$ are primitive. In the exact setting this is clearly without loss of generality, since non-zero elements of $\mathbb{R}(t)$ are units (and hence we can multiply and divide on the left by them). For approximate differential polynomials we must compute the approximate GCD of a number of polynomials in $\mathbb{R}[t]$. This is in itself an important research problem, but has been considered deeply in Kaltofen et al. (2006), which also contains a useful and current survey of related approximate GCD results. 


\subsection{Norms of differential polynomials}

To provide our notion of approximate differential polynomials a formal meaning we need a proper definition of the norm of a differential polynomial. For this we will use the coefficient 2-norm as follows.

\section{Definition 1.3.}

(i) For (a regular polynomial) $p=\sum_{0 \leq i \leq k} p_{i} t^{i} \in \mathbb{R}[t]$, define $\|p\|=\|p\|_{2}=$ $\left(\sum_{0 \leq i \leq k} p_{i}^{2}\right)^{1 / 2}$.

(ii) For $f=f_{0}+f_{1} \partial+\cdots+f_{m} \partial^{m} \in \mathbb{R}[t]\left[\partial ;^{\prime}\right]$ define $\|f\|=\|f\|_{2}=\left(\sum_{0 \leq i \leq m}\left\|f_{i}\right\|^{2}\right)^{1 / 2}$.

Note that we are assuming that our coefficients are polynomials in $\mathbb{R}[t]$, and not rational functions. One could extend the definition of norm to encompass coefficients in $\mathbb{R}(t)$, but it will not be necessary in this paper.

\subsection{The approximate GCRD problem}

We can now formally state the main problem under consideration in this paper.

Problem 1.4. Given $f, g \in \mathbb{R}[t]\left[\partial ;^{\prime}\right]$, find a small $\varepsilon>0$ and $\tilde{f}, \widetilde{g} \in \mathbb{R}[t]\left[\partial ;^{\prime}\right]$ with $\|f-\widetilde{f}\|<\varepsilon\|f\|$ and $\|g-\widetilde{g}\|<\varepsilon\|g\|$ such that $\operatorname{deg}_{\partial} \operatorname{gcrd}(\widetilde{f}, \widetilde{g}) \geq 1$.

That is, we are looking for "nearby" differential polynomials, in the coefficient 2-norm, which possess a non-trivial GCRD.

\section{GCRD via Linear Algebra}

In this section we demonstrate how to reduce the computation of the GCRD to that of linear algebra over $\mathbb{R}(t)$, and then over $\mathbb{R}$ itself. This approach has been used in the exact computation of GCRDs (see (Li and Nemes, 1997)) and Hermite forms (Giesbrecht and Kim, 2013), and has the benefit of reducing differential, and more general Ore problems, to a system of equations over a commutative field. Here we will show that it makes our approximate version of the GCRD problem amenable to numerical techniques. 


\subsection{Reduction to linear algebra over $\mathbb{R}(t)$}

Let $f, g \in \mathbb{R}(t)\left[\partial ;^{\prime}\right]$ have degrees in $\partial$ of $m$ and $n$ respectively. Then by Lemma $1.1, \operatorname{deg}_{\partial} \operatorname{gcrd}(f, g) \geq 1$ if and only if there exists $u, v \in \mathbb{R}(t)\left[\partial ;^{\prime}\right]$ such that $\operatorname{deg}_{\partial} u<n$ and $\operatorname{deg}_{\partial} v<m$ and $u f+v g=0$. We can encode the existence of $u, v$ as an $(m+n) \times(m+n)$ matrix over $\mathbb{R}(t)$ as follows. For convenience define the matrix

$$
V=V(f, g)=\left(\begin{array}{c}
\Psi_{m+n}(f) \\
\Psi_{m+n}(\partial f) \\
\vdots \\
\Psi_{m+n}\left(\partial^{n-1} f\right) \\
\Psi_{m+n}(g) \\
\Psi_{m+n}(\partial g) \\
\vdots \\
\Psi_{m+n}\left(\partial^{m-1} g\right)
\end{array}\right) \in \mathbb{R}(t)^{(m+n) \times(m+n)}
$$

the differential Sylvester matrix of $f$ and $g$ (see (Li and Nemes, 1997)), analogous to the Sylvester matrix for usual polynomials (see, e.g., (von zur Gathen, 2003, Chapter 6)).

The utility of this comes in the following observation. Let

$$
u=\sum_{0 \leq i \leq n-1} u_{i} \partial^{i}, \quad v=\sum_{0 \leq i \leq m-1} v_{i} \partial_{i} \in \mathbb{R}(t)\left[\partial ;^{\prime}\right]
$$

and

$$
w=\left(u_{0}, u_{1}, \ldots, u_{n-1}, v_{0}, v_{1}, \ldots, v_{m-1}\right) \in \mathbb{R}(t)^{1 \times(m+n)} .
$$

Then $u f+v g=0$ implies $w V=0$. This means that $w$ is a non-trivial vector in the nullspace of $V$, and in particular, $V$ is singular. Clearing denominators of $f$ and $g$ we may assume that $u, v \in \mathbb{R}[t]\left[\partial ;^{\prime}\right]$, i.e., they have polynomial coefficients, which implies that $V \in \mathbb{R}[t]^{(m+n) \times(m+n)}$. Moreover, if $f, g \in$ $\mathbb{R}[t]\left[\partial ;^{\prime}\right]$ have degrees in $t$ at most $d$ then $\operatorname{deg}_{t} V_{i j} \leq d$.

Lemma 2.1. Suppose $f, g \in \mathbb{R}[t]\left[\partial ;^{\prime}\right]$, where $\operatorname{deg}_{\partial} f=m, \operatorname{deg}_{\partial} g=n$ and $\operatorname{deg}_{t} f \leq d$ and $\operatorname{deg}_{t} g \leq d$.

- $V=V(f, g)$ is singular if and only $\operatorname{deg}_{\partial} \operatorname{gcrd}(f, g) \geq 1$.

- $\operatorname{deg}_{\partial} \operatorname{gcrd}(f, g)=\operatorname{dim}_{\operatorname{null}}(V)$, where $\operatorname{null}_{\ell}(V)$ is the left nullspace of $V$. 
- For any $w=\left(u_{0}, \ldots, u_{n-1}, v_{0}, \ldots, v_{m-1}\right) \in \mathbb{R}(t)^{1 \times(m+n)}$ such that $w V=$ 0 , we have $u f+v g=0$, where $u=\sum_{0 \leq i<n} u_{i} \partial^{i}$ and $v=\sum_{0 \leq i<m} v_{i} \partial^{i}$.

- Suppose that $\operatorname{deg}_{\partial} \operatorname{gcrd}(f, g) \geq 1$. Then there exists a $w \in \mathbb{R}[t]^{1 \times(m+n)}$ such that $w V=0$ and $\operatorname{deg}_{t} w \leq \mu=2(m+n) d$.

Proof. Part (i) - (iii) follow from Lemma 1.1 and the discussion above. Part (iv) follows from an application of Cramer's rule, and a bound on the degree of the determinants of a polynomial matrix.

Example 2.2. Let

$$
f=\partial^{2}+(0.5 t+1.0) \partial+0.3 t+0.06 t^{2}+0.2
$$

and

$$
g=\partial^{2}+\left(0.9 t^{2}+1.0+0.2 t\right) \partial+0.2+0.9 t^{2}+0.18 t^{3} .
$$

The corresponding differential Sylvester matrix $V$ is given by

$$
\left(\begin{array}{cccc}
0.3 t+0.06 t^{2}+0.2 & 0.5 t+1.0 & 1 & 0 \\
0.3+0.12 t & 0.7+0.06 t^{2}+0.3 t & 0.5 t+1.0 & 1 \\
0.2+0.9 t^{2}+0.18 t^{3} & 0.9 t^{2}+1.0+0.2 t & 1 & 0 \\
1.8 t+0.54 t^{2} & 0.9 t^{2}+0.18 t^{3}+1.8 t+0.4 & 0.9 t^{2}+1.0+0.2 t & 1
\end{array}\right)
$$

$V$ has rank 3 with the (left) null space vector

$$
\left(\begin{array}{c}
-27.0 t^{4}+9.0 t^{3}+60.0 t-10.0 \\
-30.0 t^{2}+10.0 t \\
9.0 t^{3}-3.0 t^{2}-60.0 t+10.0 \\
30.0 t^{2}-10.0 t
\end{array}\right)^{T}
$$

Definition 2.3. For any matrix $V \in \mathbb{R}(t)\left[\partial ;^{\prime}\right]$, we define the Frobenius norm $\|V\|_{F}$ by

$$
\|V\|_{F}^{2}=\sum_{i j}\left\|V_{i j}\right\|^{2}
$$

Lemma 2.4. Let $f, g \in \mathbb{R}[t]\left[\partial ;^{\prime}\right]$ have $\operatorname{deg}_{\partial} f \leq m$, $\operatorname{deg}_{\partial} g \leq n$, and both have degree in $t$ at most d. Let $V=V(f, g)$ be the differential Sylvester matrix of $f$ and $g$. Then $\|V\|_{F}^{2} \leq d^{2 n}\|f\|^{2}+d^{2 m}\|g\|^{2}$. 
Proof. First note that $\|\partial f\|^{2} \leq\left(d^{2}+1\right)\|f\|^{2}$, and hence $\left\|\partial^{k} f\right\|^{2} \leq\left(d^{2}+\right.$ 1) ${ }^{k}\|f\|^{2}$. Thus

$$
\begin{aligned}
\|V\|_{F}^{2} & =\sum_{0 \leq i<n}\left\|\partial^{i} f\right\|^{2}+\sum_{0 \leq i<m}\left\|\partial^{i} g\right\|^{2} \\
& \leq \sum_{0 \leq i<n}\left(d^{2}+1\right)^{i}\|f\|^{2}+\sum_{0 \leq i<m}\left(d^{2}+1\right)^{i}\|g\|^{2} \\
& \leq d^{2 n}\|f\|^{2}+d^{2 m}\|g\|^{2} .
\end{aligned}
$$

Note that the exponentials of $d$ (or, more precisely, the falling factorials) are intrinsic in the resultant formulation, but will cause considerable numerical instability for large degrees in $\partial$. As is typical with differential polynomials we generally restrict ourselves to small degrees in $\partial$.

\subsection{Reduction to linear algebra over $\mathbb{R}$}

Next we show how to encode the existence of a GCRD as a linear algebra problem over $\mathbb{R}$, as opposed to $\mathbb{R}(t)$. Again let $V \in \mathbb{R}[t]^{(m+n) \times(m+n)}$ be the differential Sylvester matrix of $f, g \in \mathbb{R}[t]\left[\partial ;^{\prime}\right]$ of degrees $m$ and $n$ respectively in $\partial$, and degrees at most $d$ in $t$. From Lemma 2.1 we know that if a GCRD exists then there is a $w \in \mathbb{R}[t]^{1 \times(m+n)}$ such that $w V=0$, with $\operatorname{deg}_{t} w \leq \mu=$ $2(m+n) d$.

Now suppose $b=b_{0}+b_{1} t+\cdots+b_{\mu+d} t^{\mu+d} \in R[t]$ and define

$$
\Psi(b)=\left(b_{0}, b_{1}, \ldots, b_{\mu+d}\right) \in \mathbb{R}^{1 \times(\mu+d+1)} .
$$

For any polynomial $a \in \mathbb{R}[t]$ of degree at most $d$ let

$$
\Gamma(a)=\left(\begin{array}{c}
\Psi(a) \\
\Psi(t a) \\
\vdots \\
\Psi\left(t^{\mu} a\right)
\end{array}\right) \in \mathbb{R}^{(\mu+1) \times(\mu+d+1)}
$$

$\Gamma(a)$ is the left multiplier matrix of $a$ with respect to the basis $\left\langle 1, t, \ldots, t^{\mu+d}\right\rangle$.

Now given the $(m+n) \times(m+n)$ matrix $V$ we apply $\Gamma$ entry-wise to $V$ to obtain $\widehat{V} \in \mathbb{R}^{(m+n)(\mu+1) \times(m+n)(\mu+d+1)}$; each entry of $V$ in $\mathbb{R}[t]$ is mapped to a block entry $\mathbb{R}^{(\mu+1) \times(\mu+d+1)}$ in $\widehat{V}$. We refer to $\widehat{V}$ as the inflated differential Sylvester matrix of $f$ and $g$. 
Lemma 2.5. Let $f, g \in \mathbb{R}[t]\left[\partial ;^{\prime}\right]$ be as above, with differential Sylvester matrix $V \in \mathbb{R}[t]^{(m+n) \times(m+n)}$ and inflated differential Sylvester matrix $\widehat{V} \in$ $\mathbb{R}^{(m+n)(\mu+1) \times(m+n)(\mu+d+1)}$. There exists a $w \in \mathbb{R}[t]^{1 \times(m+n)}$ such that $w V=0$, if and only if there exists a $\widehat{w} \in \mathbb{R}^{(\mu+d+1) \times(m+n)(\mu+1)}$ such that $\widehat{w} \widehat{V}=0$. More generally,

$$
\operatorname{deg}_{\partial} \operatorname{gcrd}(f, g)=\frac{\operatorname{dim} n u l l_{\ell}(\widehat{V})}{\mu+d+1} .
$$

Proof. This follows directly from the definition of $\Gamma$ and Lemma 2.1.

Example 2.6. Consider $f=(0.84 t+0.45) \partial+0.11 t+0.42$ and $g=0.66 \partial+$ $0.92 t$. Then the matrix $\widehat{V}(f, g)$ is given by

$$
\left(\begin{array}{cccccccccccc}
0.42 & 0.11 & 0 & 0 & 0 & 0 & 0.45 & 0.84 & 0 & 0 & 0 & 0 \\
0 & 0.42 & 0.11 & 0 & 0 & 0 & 0 & 0.45 & 0.84 & 0 & 0 & 0 \\
0 & 0 & 0.42 & 0.11 & 0 & 0 & 0 & 0 & 0.45 & 0.84 & 0 & 0 \\
0 & 0 & 0 & 0.42 & 0.11 & 0 & 0 & 0 & 0 & 0.45 & 0.84 & 0 \\
0 & 0 & 0 & 0 & 0.42 & 0.11 & 0 & 0 & 0 & 0 & 0.45 & 0.84 \\
0 & 0.92 & 0 & 0 & 0 & 0 & 0.66 & 0 & 0 & 0 & 0 & 0 \\
0 & 0 & 0.92 & 0 & 0 & 0 & 0 & 0.66 & 0 & 0 & 0 & 0 \\
0 & 0 & 0 & 0.92 & 0 & 0 & 0 & 0 & 0.66 & 0 & 0 & 0 \\
0 & 0 & 0 & 0 & 0.92 & 0 & 0 & 0 & 0 & 0.66 & 0 & 0 \\
0 & 0 & 0 & 0 & 0 & 0.92 & 0 & 0 & 0 & 0 & 0.66 & 0
\end{array}\right)
$$

We can now bound the norm of the inflated differential Sylvester matrix.

Lemma 2.7. Let $f, g \in \mathbb{R}[t]\left[\partial ;^{\prime}\right]$ have $\operatorname{deg}_{\partial} f \leq m$, $\operatorname{deg}_{\partial} g \leq n$ and both have degree degree at most $d$ in $t$. Let $\widehat{V} \in \mathbb{R}^{(m+n)(\mu+1) \times(m+n)(\mu+d+1)}$ be the inflated differential Sylvester matrix of $f$ and $g$, where $\mu=2(m+n) d$. Then $\|\widehat{V}\|_{2} \leq \mu \cdot\left(d^{2 n}\|f\|^{2}+d^{2 m}\|g\|^{2}\right)$.

Proof. Each row of $\widehat{V}$ consists precisely of entries of $V=V(f, g)$, shifted in position with respect to the previous row. Thus

$$
\begin{aligned}
& \|\widehat{V}\|_{F}^{2} \leq \mu \cdot\|V\|_{F}^{2} \leq \mu \cdot\left(d^{2 n}\|f\|^{2}+d^{2 m}\|g\|^{2}\right), \text { and } \\
& \|\widehat{V}\|_{2}^{2} \leq\|\widehat{V}\|_{F}^{2} .
\end{aligned}
$$

See (Golub and van Loan, 2013, §2.3.2). 


\section{Computing an approximate GCRD}

We have now formulated the problem of determining the existence of GCRD's of differential polynomials in $\mathbb{R}[t]\left[\partial ;^{\prime}\right]$ as one of computing left null vectors of the inflated differential Sylvester matrix over $\mathbb{R}$. We can now adapt the approach of Corless et al. (1995) of using the SVD to find the nearest singular matrix. While this will not be perfect, in that the nearest singular matrix will not generally have the same structure as the inflated differential Sylvester matrix, if our input differential polynomials are "nearby" polynomials with a non-trivial GCRD we will generally recover them.

For convenience we will generally assume throughout this section that our input differential polynomials are normalized, that is have coefficient 2-norm 1 under the definition of Section 1.2. This can, of course, be enforced by a simple a priori renormalization, i.e., dividing through by the actual norm, and does not affect the generality or quality of the results.

\subsection{Finding nearby non-trivial GCRDs}

It is well understood how to find the nearest singular unstructured matrix to a given matrix via the singular value decomposition (SVD); see (Golub and van Loan, 2013, §8.6). We will assume in this section that $V \in \mathbb{R}[t]^{N \times N}$ is the differential Sylvester matrix from Subsection 2.1, of differential polynomials $f, g \in \mathbb{R}[t]\left[\partial ;^{\prime}\right]$ of degrees (in $\partial$ ) of $m$ and $n$ respectively, with $N=m+$ $n$. From this we construct the inflated differential Sylvester matrix $\widehat{V} \in$ $\mathbb{R}^{N(\mu+1) \times N(\mu+d+1)}$ as in Subsection 2.2. Using the SVD we can find the matrix $\Delta \widehat{V}$ of minimal 2-norm such that $\widehat{V}+\Delta \widehat{V}$ has a prescribed rank. First, we compute the SVD of $\widehat{V}$ as

$$
\widehat{V}=P \Sigma Q
$$

where

$$
P \in \mathbb{R}^{N(\mu+1) \times N(\mu+1)}, \text { and } Q \in \mathbb{R}^{N(\mu+d+1) \times N(\mu+d+1)}
$$

are orthogonal and

$$
\Sigma=\operatorname{diag}\left(\sigma_{1}, \ldots, \sigma_{N(\mu+1)}\right) \in \mathbb{R}^{N(\mu+1) \times N(\mu+d+1)},
$$

satisfies $\sigma_{1} \geq \sigma_{2} \ldots \geq \sigma \geq \sigma_{N(\mu+1)}$. Note that $\Sigma$ is not square (it has more columns than rows), and we simply pad it with zeros to obtain the desired shape. 
Now, by Lemma 2.5, we want to find a nearby matrix whose left nullspace has reduced dimension by multiples of $(\mu+d+1)$, that is

$$
P \bar{\Sigma} Q=\widehat{V}+\Delta \widehat{V}
$$

where

$$
\begin{aligned}
\bar{\Sigma} & =\operatorname{diag}\left(\sigma_{1}, \sigma_{2}, \ldots, \sigma_{(N-\varrho)(\mu+d+1)}, 0, \ldots, 0\right) \\
& \in \mathbb{R}^{N(\mu+1) \times N(\mu+d+1)},
\end{aligned}
$$

where $\varrho=\frac{\operatorname{dim} \operatorname{null}_{\ell}(\widehat{V}+\Delta \widehat{V})}{\mu+d+1}$. Then $\widehat{V}$ will be by the singular matrix, $\widehat{V}+\Delta \widehat{V}$ of prescribed rank. Of course, $\widehat{V}+\Delta \widehat{V}$ is probably an unstructured matrix, and in particular, not an inflated differential Sylvester matrix.

Next we show that a matrix of the desired rank deficiency and (inflated differential) structure exists within a relatively small radius of $\widehat{V}$. Suppose there is an $\widetilde{f}, \widetilde{g} \in \mathbb{R}[t]\left[\partial ; ;^{\prime}\right]$, with $\|\widetilde{f}-f\| \leq \varepsilon$ and $\|\widetilde{g}-g\| \leq \varepsilon$, such that $\operatorname{deg}_{\partial} \operatorname{gcrd}(\widetilde{f}, \widetilde{g})=\varrho \geq 1$. Let $\Delta f=f-\widetilde{f}$ and $\Delta g=g-\widetilde{g}$, so $\|\Delta f\|,\|\Delta g\|<\varepsilon$. Moreover, the differential resultant matrix $W \in \mathbb{R}[t]\left[\partial ; ;^{\prime}\right]^{N \times N}$ formed from $\Delta f$, and $\Delta g$ has

$$
\|W\|_{F}^{2}<\left(d^{2 n}+d^{2 m}\right) \cdot \varepsilon^{2}
$$

by Lemma 2.4. Thus, the inflated differential resultant matrix $\widehat{W} \in \mathbb{R}^{N(\mu+1) \times N(\mu+d+1)}$ has

$$
\|\widehat{W}\|_{2}^{2}<\mu \cdot\left(d^{2 n}+d^{2 m}\right) \cdot \varepsilon^{2}
$$

by Lemma 2.7. Moreover, $\operatorname{dim}_{n u l l}(\widehat{V}+\widehat{W})=\varrho(\mu+d+1)$. Thus, for sufficiently small $\varepsilon$ there exists a perturbation $\widehat{W}$ such that $\|\widehat{W}\|$ is small (at least assuming small $n, m$ ) and $\widehat{V}+\widehat{W}$ has appropriate rank and structure.

Due to the unstructured nature of $\widehat{V}+\Delta \widehat{V}$, one must take care in working with a reasonable approximation for $f$ and $g$. However, there is considerable redundancy of the coefficients of $f$ and $g$ in their inflated differential Sylvester matrix, if only because each entry of $f$ and $g$ shows up multiple times under the map $\Gamma$; see Section 2.2. There is, in fact, even more redundancy because of the different derivatives in rows of the differential Sylvester matrix, but we will not capitalize on this. 


\subsection{Computing $V+\Delta V$ and reconstructing $f+\Delta f$ and}

$$
g+\Delta g
$$

To form $V+\Delta V$ we take a weighted average of the $t$-shifted blocks of $\widehat{V}+\Delta \widehat{V}$ that correspond to $f$ and $g$. This involves identifying the "blocks" of $\widehat{V}+\Delta \widehat{V}$ that correspond to $f$ and $g$ and re-constructing them entrywise ensuring the entries in degree $t$ of $f$ and $g$ do not increase. The $f$ block consists of rows 1 through $\mu+1$ and the $g$ block consists of rows $\operatorname{deg}_{\partial} g(\mu+1)+1$ through $\operatorname{deg}_{\partial} g(\mu+1)+\mu+2$. The columns in both cases are the $\mu+d+1$ columns for each block entry.

The reason that this reconstruction is often satisfactory is we have that if $\varrho>0$ then $\widehat{V}$ has $\operatorname{rank}(N-\varrho)(\mu+d+1)$, and if $\sigma_{(N-\varrho)(\mu+d+1)+1}<\varepsilon$, then

$$
\begin{aligned}
\|\Sigma-\bar{\Sigma}\|_{F}^{2} & =\sum_{i=(N-\varrho)(\mu+d+1)+1}^{(\mu+1) N}\left|\sigma_{i}\right|^{2} \\
& \leq \varepsilon^{2}[(\mu+1) N-(N-\varrho)(\mu+d+1)] \\
& \leq \varepsilon^{2} \varrho(\mu+d+1) .
\end{aligned}
$$

We have that $\|\widehat{W}\|_{F}^{2}=\|\Sigma-\bar{\Sigma}\|_{F}^{2}$ (Golub and van Loan, 2013, Corollory 2.4.3) because the singular values of $\widehat{W}=P(\Sigma-\bar{\Sigma}) Q$ are a permutation of the entries along the main diagonal of $\Sigma-\bar{\Sigma}$.

In our construction we require that $\operatorname{deg}_{t} \widetilde{f}_{i} \leq \operatorname{deg}_{t} f_{i}$ for $0 \leq i \leq \operatorname{deg}_{\partial} f$ and a similar condition on $g$ in order to preserve the structure of $V+\Delta V$. Furthermore, if the perturbation from adjusting the singular values is small, then the non-zero entries are "small" and can usually be ignored without losing too much information.

We should now have a matrix that is numerically singular, $V+\Delta V$ and perturbations $\Delta f$ and $\Delta g$ such that NumericGCRD $(f+\Delta f, g+\Delta g)$ is non trivial and satisfies the conditions $\operatorname{deg}_{t} \Delta f_{i} \leq \operatorname{deg}_{t} f_{i}$ for $0 \leq i \leq m$ and $\operatorname{deg}_{t} \Delta g_{j} \leq \operatorname{deg}_{t} g_{j}$ for $0 \leq j \leq n$.

\subsection{Computing the Approximate GCRD}

Let $f, g \in \mathbb{R}[t]\left[\partial ; ;^{\prime}\right]$ have degrees $m$ and $n$ respectively. Let $G=\operatorname{gcrd}(f, g)$ and $\operatorname{deg}_{\partial}(G)=D$. Then one may obtain an $\mathbb{R}(t)$ multiple of $G$ by solving

$$
w V=\left(\begin{array}{lllllll}
*_{0} & *_{1} & \cdots & *_{D} & 0 & \cdots & 0
\end{array}\right),
$$


where we do not care about the entry $*$. Solving this system will give us a multiple of $G$, which we may assume is in $\mathbb{R}[t]$ by clearing fractions from the denominator.

\section{Computing an Approximate Primitive GCRD}

When computing the GCRD numerically we obtain a result that is an $\mathbb{R}[t]$ multiple of a primitive GCRD upon clearing fractions. In some applications it is desirable to remove this content. However the coefficients are not exactly known so taking an exact GCD of the coefficients will yield unsatisfactory answers.

Consider the case of our NumericGCRD algorithm, Algorithm 2. Our solution will have approximate content even if we use rational arithmetic because $f+\Delta f$ and $g+\Delta g$ have an approximate GCRD but may not have a GCRD algebraically due to round off errors in their recovery. If a primitive solution is desired, then techniques to remove the content are required.

\section{Leading Coefficient Known in Advance}

Since leading coefficients of GCRDs are propagated through multiplication, we often know the leading coefficient of a GCRD in advance. In general, the leading coefficient of the GCRD should be approximately one (i.e., a constant) save a few special cases where the leading coefficients of $f$ and $g$ have a non-trivial approximate GCD.

As an observation, given $f, g$ in $\mathbb{R}[t]\left[\partial ;^{\prime}\right]$ where $\operatorname{deg}_{\partial} f=m$ and $\operatorname{deg}_{\partial} g=n$ and $\operatorname{gcd}\left(f_{m}, g_{n}\right)=1$ then for a suitable approximate GCD algorithm (see Corless et al. (2004) or Zeng and Dayton (2004)) we have that a primitive numeric GCRD of $f$ and $g$ satisfies $\operatorname{lcoeff}(G)=1$. The reason that we need the notion of approximate GCD is that it is possible that we may have different algorithms returning different answers. Consider $t^{2}$ and $t^{2}+2^{-s}$ where $s$ is large. Algebraically both polynomials are co-prime but if $s$ is sufficiently large then some GCD algorithms will return a non trivial GCD. To justify this, let $\operatorname{deg}_{\partial} G=D$. $G$ is a GCRD so we have that $G$ divides both $f$ and $g$ on the right. If $G$ is primitive, it follows that $G_{D} \mid f_{m}$ and $G_{D} \mid g_{n}$ so $G_{D} \mid \operatorname{gcd}\left(f_{m}, g_{n}\right)=1$. This occurs if and only if $G_{D}=1$.

If we are given a candidate GCRD $\widetilde{G}$ that is not primitive, where $\operatorname{deg}_{\partial} \widetilde{G}=$ $D$, and we know the primitive GCRD has leading coefficient 1 , then lcoeff $(\widetilde{G}) \approx$

cont $\widetilde{G}$. It follows that $\widetilde{G}_{D} \mid \widetilde{G}_{i}$ for $0 \leq i<D$. This means that the remainders 
are numerically trivial, so we can assume they are zero. Using a method of approximate division we can recover a primitive approximate GCRD.

If the primitive GCRD is known to be 1 , it is not sufficient to solve the system

$$
w V=\left(\begin{array}{lllllll}
*_{0} & *_{1} & \ldots & *_{D-1} & 1 & 0 \ldots 0
\end{array}\right)^{T}
$$

because we are performing numerical linear algebra and we will often obtain a solution over $\mathbb{R}(t)\left[\partial ;^{\prime}\right]$. One particular method of approximate division is by interpolation. This particular method of approximate division yields answers that one would expect with prior knowledge of a GCRD and a uniform distribution of noise. As expected with an interpolation based method, it will break down if we are unable to accurately compute the degrees of terms due to artifacts and round off errors. The method of Bini and Pan (1986) for approximate division via a Fast Fourier Transform (FFT) is used for content removal in this case, and proves both fast and numerically robust in practice.

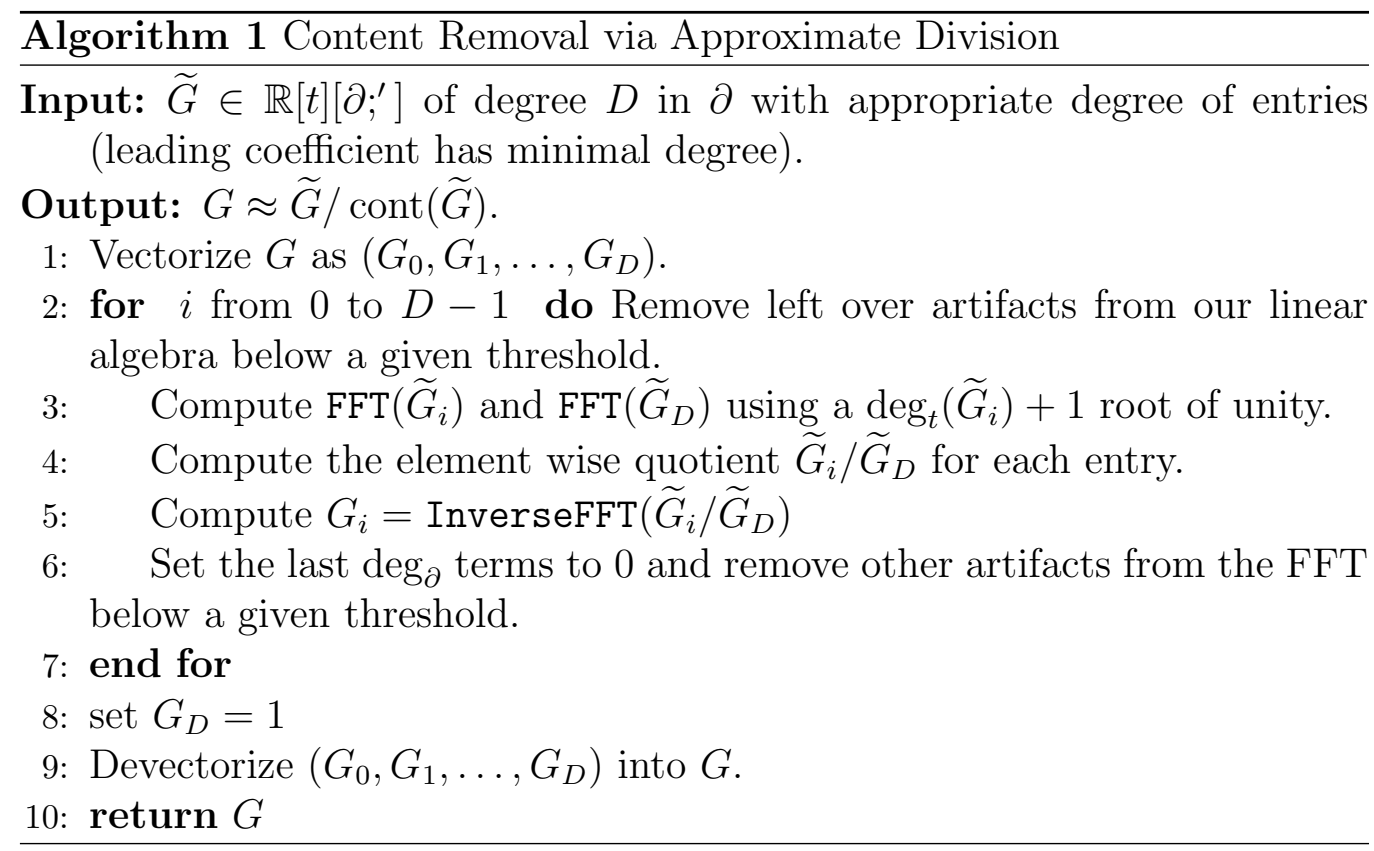

Example 3.1 (Numeric GCRD). Consider

$$
f=-0.45 \partial^{2}-0.56 t \partial-0.11 t^{2}-0.45
$$

and

$$
g=\partial^{3}+(t+0.66) \partial^{2}+(2.0+0.952 t) \partial+0.66+0.292 t^{2}
$$


A numeric GCRD of $f$ and $g$ is given by

$$
\begin{aligned}
& G=\left(0.02781 t^{2}+0.30990 t-0.01460 t^{3}-0.11380\right) \partial \\
& +0.02781 t^{3}+0.30993 t^{2}-0.01461 t^{4}-0.11378 t-0.00002 .
\end{aligned}
$$

Given the low degrees and leading coefficients, a primitive GCRD is probably a unit in $\mathbb{R}$. Removing content with an FFT gives us a primitive numeric GCRD of $\partial+1.00000 t$.

\section{Leading Coefficient of $G$ Unknown}

It is not possible to determine the leading coefficient of a primitive GCRD in advance when the leading coefficients of $f$ and $g$ have a non-trivial GCD, or if they share a nearby common solution. In order work around this one would need to approximate $\operatorname{cont}(G)=\operatorname{gcd}\left(G_{0}, G_{1}, \ldots, G_{D}\right)$ numerically, then perform an approximate polynomial division. We used the method of Corless et al. (2004) to compute pair-wise GCDs and obtained somewhat mixed results. In some instances the content had a degree that was too small based on our construction or our division algorithm did not provide an answer consistent with the GCRD we constructed. We might hope to overcome some of these problems using a more specific method for the GCD of multiple polynomials, as developed in Kaltofen et al. (2005). 


\subsection{Algorithms}

This section provides a high-level description of the primary algorithms we are using, in pseudocode.

In practice, we will demonstrate that our algorithms work well on low degree differential polynomials as input. However, in general our algorithms are not provably guaranteed to give close polynomials with a non-trivial GCRD. In practice, if $\|f\|=\|g\|=1$ and $\|\Delta f\|=\|\Delta g\|<0.1$ then for low degree differential polynomials we are usually able to get an answer that is acceptable based on our prior knowledge of $\operatorname{gcrd}(f, g)$.

\section{Algorithm 2 : NumericGCRD}

\section{Input:}

- $f, g \in \mathbb{R}[t]\left[\partial ;^{\prime}\right]$ non-zero with $\|f\|=\|g\|=1$;

- A search radius $\varepsilon>0$.

Output: $G \approx \operatorname{gcrd}(f, g) \in \mathbb{R}[t]\left[\partial ;^{\prime}\right]$ with $\operatorname{deg}_{\partial} G \geq 1$, or an indication that $f$ and $g$ are co-prime within search radius $\varepsilon$.

1: $m \leftarrow \operatorname{deg}_{\partial} f, n \leftarrow \operatorname{deg}_{\partial} g, d \leftarrow \max \left\{\operatorname{deg}_{t} f, \operatorname{deg}_{t} g\right\}$ and $\mu \leftarrow 2(m+n) d$.

2: Form the differential Sylvester matrix $V(f, g) \in \mathbb{R}[t]^{(m+n) \times(m+n)}$.

3: Form the inflated differential Sylvester matrix $\widehat{V}=\widehat{V}(f, g) \in \mathbb{R}^{(m+n)(\mu+1) \times(m+n)(\mu+d+1)}$ of $V$.

4: Compute the numerical rank $r$ of $V$ using Algorithm 4 on $\widehat{V}$ with search radius $\varepsilon$.

5: If $r>0$ then set $D=m+n-r$. Otherwise indicate that $f$ and $g$ are co-prime with respect to $\varepsilon$ and return.

6: Solve for $w$ from

$$
w V=\left(\begin{array}{lllllll}
*_{0} & *_{1} & \ldots & *_{D} & 0 & \ldots & 0
\end{array}\right)^{T} .
$$

7: Set $G=w V$

8: Optionally remove the content from $G$ numerically.

As an observation, for $f, g \in \mathbb{R}[t]\left[\partial ;^{\prime}\right]$ of degrees $m$ and in $n$ in $\partial$ respectively, we can use some heuristics to detect failures of our algorithms. Let $G=\operatorname{gcrd}(f, g)$ and $\operatorname{deg}_{\partial} G=D$. It is clear that if $D>\min \{n, m\}$ then $G$ cannot be a GCRD. Simmilarily if $\operatorname{deg}_{t}\left(G_{i}\right)<\operatorname{deg}_{t}\left(G_{D}\right)$ for some $0 \leq i<D$ then $G$ cannot be a GCRD of $f$ and $g$ over $\mathbb{R}[t]$ if $\operatorname{gcd}(\operatorname{lcoeff}(f), \operatorname{lcoeff}(g))=1$. 


\section{Algorithm 3 : Nearest With GCRD}

\section{Input:}

- $f, g \in \mathbb{R}[t]\left[\partial ;^{\prime}\right]$ with $\|f\|=\|g\|=1$;

- A search radius $\varepsilon>0$.

Output: $f+\Delta f, g+\Delta g$ where $\operatorname{deg}_{t} \Delta f \leq \operatorname{deg}_{t} f, \operatorname{deg}_{\partial} \Delta f \leq \operatorname{deg}_{\partial} f$, $\operatorname{deg}_{t} \Delta g \leq \operatorname{deg}_{t} g, \operatorname{deg}_{\partial} \Delta g \leq \operatorname{deg}_{\partial} g$ and

$G \approx \operatorname{gcrd}(f+\Delta f, g+\Delta g) \in \mathbb{R}[t]\left[\partial ;^{\prime}\right]$ with $\operatorname{deg}_{\partial} G \geq 1$, or an indication that $f$ and $g$ are co-prime within search radius $\varepsilon$.

1: $m \leftarrow \operatorname{deg}_{\partial} f, n \leftarrow \operatorname{deg}_{\partial} g, d \leftarrow \max \left\{\operatorname{deg}_{t} f, \operatorname{deg}_{t} g\right\}$ and $\mu \leftarrow 2(m+n) d$.

2: Form the differential Sylvester matrix $V(f, g) \in \mathbb{R}[t]^{(m+n) \times(m+n)}$.

3: Form the inflated differential Sylvester matrix

$\widehat{V}=\widehat{V}(f, g) \in \mathbb{R}^{(m+n)(\mu+1) \times(m+n)(\mu+d+1)}$ of $V$.

4: Compute the SVD of $\widehat{V}, \widehat{V}=P \Sigma Q$ with $P, \Sigma$ and $Q$ as discussed in $\S 3.1$.

5: Compute the numerical rank $r$ of $V$ using Algorithm 4 on $\widehat{V}$ with search radius $\varepsilon$.

6: If $r>0$ set the last $r(\mu+d+1)$ singular values to 0 and compute $\bar{\Sigma}$ as discussed in $§ 3.1$. Otherwise indicate that $f$ and $g$ are co-prime with respect to $\varepsilon$ and return.

7: Compute $\widehat{V}+\Delta \widehat{V}=P \bar{\Sigma} Q$.

8: Recover $f+\Delta f$ and $g+\Delta g$ from $\widehat{V}+\Delta \widehat{V}$ as discussed in $\S 3.2$.

9: Compute $G=\operatorname{NumericGCRD}(f+\Delta f, g+\Delta g)$ using Algorithm 2 with $\varepsilon$ used to validate the degree of our approximate GCRD.

10: Optionally remove the content from $G$ numerically.

Example 3.2 (Nearest With GCRD). Consider

$$
f=(1.0+0.0043 t) \partial^{2}+(3.0 t-0.0003) \partial+2.0 t^{2}+1.0
$$

and

$$
g=t^{2} \partial^{2}+\left(-0.0004 t+t^{3}+0.0001\right) \partial+t^{2}
$$

with a given search radius $\varepsilon=.5 \cdot 10^{-2}$. Applying Algorithm 4 on the singular values of $\widehat{V}$ we obtain a rank of 78 where the expected rank is 84 , which is within reason. From this we conclude that the degree of our GCRD is 1 and 


\section{Algorithm 4 : Deflated Rank}

\section{Input:}

- An inflated differential Sylvester matrix

$\widehat{V} \in \mathbb{R}^{(m+n)(\mu+1) \times(m+n)(\mu+d+1)}$ with $m, n, d$ and $\mu$ defined as in Algorithm 2 or Algorithm 3.

- A search radius $\varepsilon>0$

Output: The the numeric rank of the (non-inflated) differential Sylvester matrix $V$ from Algorithm 2 or 3.

1: Find the maximum $k$ such that $\sigma_{k}>\varepsilon \frac{\sqrt{(m+n)(2 \mu+d+2)}}{\mu+d+1}$ and $\sigma_{k+1}<\varepsilon$.

2: if $\sigma_{k}>\varepsilon$ for all $k$ then $\widehat{V}$ has full rank.

3: If there is no significant change between $\sigma_{k}$ and $\sigma_{k+1}$ for all $k$ as determined by step 2 then return failure.

4: Set $r=\left\lceil\frac{k}{\mu+d+1}\right\rceil$, the scaled rank.

we compute

$$
\begin{aligned}
\tilde{f} & =0.99999+2.00000 t^{2} \\
& +(-0.00029+3.00000 t) \partial \\
& +(0.99999+0.00429 t) \partial^{2}
\end{aligned}
$$

and

$$
\begin{aligned}
\widetilde{g} & =-0.00001-0.00002 t+1.00001 t^{2} \\
& +\left(0.00011-0.00039 t+0.00005 t^{2}+0.99999 t^{3}\right) \partial \\
& +\left(-0.00001-0.00002 t+0.99999 t^{2}\right) \partial^{2} .
\end{aligned}
$$

Furthermore, we obtain $\operatorname{NumericGCRD}(\hat{f}, \hat{g}) \approx-0.06695+1.06508 t+\partial$, after removing content. We have that the size of the perturbations are $\| f-$ $\widetilde{f} \|=1.22023 \times 10^{-9}$ and $\|g-\widetilde{g}\|=0.00007$. In this example the largest singular value we removed was known to three decimal places so we can expect the accuracy of our answer to reflect this.

As a remark, we constructed $\widetilde{f}$ and $\widetilde{g}$ from their first occurrence in $\widehat{V}$ for illustration purposes. In general weighted approaches work well and are demonstrated in Section 5. 


\section{On the Rank Algorithm}

Algorithm 4 follows similarly to the method for determination of numerical rank in the SVD GCD of Corless et al. (1995). In our circumstances the singular values are in (column) "blocks" of size $\mu+d+1$. We choose to declare the scaled rank as $\left\lceil\frac{k}{\mu+d+1}\right\rceil$ because it will tend to ignore spurious singular values provided there are fewer spurious singular values than the block size. Since we know that non-spurious singular values come in the block size, this allows us to accurately compute the rank of $V$ even if we don't correctly compute the rank of $\widehat{V}$ in the case of low degree differential polynomials.

Another important reason for this decision is if we set a singular value to 0 that is reasonably far away from the next singular value then the resulting matrix $\widehat{V}+\Delta \widehat{V}$ becomes highly unstructured and our algorithm fails to produce meaningful results.

It is possible that the singular values are not clearly separated. This happens frequently with dense large degree differential polynomials. In this scenario it is possible to under estimate the rank of $V$. If $f$ and $g$ are known to have a GCRD, then it may be possible to eliminate bad GCRD candidates using heuristics.

\section{Complexity \& Stability Analysis}

In this section we investigate the computational complexity in terms of the input size and some of the numerical stability of the algorithms. We assume as usual that for $f, g \in \mathbb{R}[t]\left[\partial ;^{\prime}\right]$ that $\operatorname{deg}_{\partial} f=m, \operatorname{deg}_{\partial} g=n, V=V(f, g)$ $\in \mathbb{R}[t]^{(m+n) \times(m+n)}$ and $\widehat{V} \in \mathbb{R}^{(m+n)(\mu+1) \times(m+n)(\mu+d+1)}$ with $\operatorname{deg} V=d$. We assume that all operations over $\mathbb{R}$ can be performed in a constant amount of time, i.e., are floating point operations or flops. Our algorithms will be analyzed in a bottom-up approach, reflecting their dependencies.

\section{Analysis of Algorithm 4: Deflated Rank}

The cost of the algorithm is dependent on computing the singular values of $\widehat{V}$. The cost of the computing the singular values of $\widehat{V}$ is $O\left((m+n)^{6} d^{3}\right)$ operations over $\mathbb{R}$ or flops, using the standard method of matrix multiplication. 


\section{Analysis of Algorithm 2: NumericGCRD}

The cost of the algorithm is dominated by that of computing the singular values of $\widehat{V}$ to determine the rank of $V$. The cost of performing the linear algebra on $V$ is $\left.O(m+n)^{3} d^{2}\right)$ operations over $\mathbb{R}$. The cost of the SVD on $\widehat{V}$ is $O\left((m+n)^{6} d^{3}\right)$ operations and hence dominates.

In general the numerical stability of the algorithms depends on the methods used to solve the problem and the conditioning of $V$. It is difficult to say much else without making further assumptions. We hope to explore this further in subsequent work.

\section{Analysis of Algorithm 3: Nearest With GCRD}

The cost of the algorithm is dominated by that of computing the singular values of $\widehat{V}$ to determine the rank of $V$. This requires $O\left(\left((m+n)^{6} d^{3}\right)\right.$ operations over $\mathbb{R}$ or flops, with the usual method of matrix multiplication. This algorithm will call Algorithm 2, but again the cost of computing the singular values of $\widehat{V}$ is the dominating cost.

The accuracy of our answer is subject to the singular values we set to zero or remove. In particular, the larger the singular values we remove, the less accurate our answer typically becomes. In our experiments this became noticeable with singular values around $10^{-3}$. Under the assumption that noise is distributed uniformly over $f$ and $g$ then the singular values of $\widehat{V}$ will generally remain small, which means Algorithm 3 typically produce a reasonable numeric GCRD. Again, we hope to bolster these heuristic observations with more careful analysis in subsequent work.

\section{Experimental Evaluation}

In order to verify the robustness of the algorithm and whether it is able to compute $\widetilde{f}$ and $\widetilde{g}$ reliably, we performed 100 random trials with different search neighborhoods and adding random amounts of noise. In our experiments we are adding a noise factor of size $\delta$ and working with a search radius of size $\rho$. The goal of our experiments is to demonstrate that given a pair of relatively prime polynomials $\widehat{f}, \widehat{g} \in \mathbb{R}[t]\left[\partial ;^{\prime}\right]$ where $\|f-\widehat{f}\|=\|g-\widehat{g}\|=\delta$ for some $f$ and $g$ such that $\operatorname{gcrd}(f, g)$ is non trivial, we can recover a pair of polynomials such that Algorithm 3 returns a non trivial answer in a given search radius of size $\rho$. More precisely, the perturbations $\|\widehat{f}-\widetilde{f}\|$ 
and $\|\widehat{g}-\widetilde{g}\|$ are minimal approximations to the 2-norm we are using in our least squares setting. We can think of $\varepsilon$ from Problem 1.4 as being quantified by $\max \{\|\widehat{f}-\widetilde{f}\|,\|\widehat{g}-\widetilde{g}\|\}$ in these tests.

We perform tests on two sets of examples where the input size was bounded. We found the algorithm worked quite well in practice on examples taken uniformly at random, however the lack normalization or structure made it difficult to obtain comparable data. In the data tables we provide statistics for two different reconstructions. The first approach is reconstructing $\widetilde{f}$ and $\widetilde{g}$ from the first row they appear in $\widehat{V}+\Delta \widehat{V}$. The other reconstruction approach is using the weighted approach over the entire block to recover $\widetilde{f}_{w}$ and $\widetilde{g}_{w}$ respectively. On average the weighted approach tends to smooth values over at the cost of structure where as taking the first row can preserve the underlying structure of $f$ and $g$, especially if they are sparse.

We note that as the noise decreases so does the size of the perturbation. We will continue to get smaller perturbations until the roundoff error from writing $\widehat{V} \approx P \Sigma Q$ dominates the perturbation sizes. If the noise is sufficiently small then we are executing NumericGCRD (Algorithm 2) as the perturbations will become indistinguishable from roundoff errors. Despite the noise in some tests appearing large, it is distributed uniformly into the coefficients resulting in each coefficient being perturbed by a fraction of the total amount of noise added. Although the worst case perturbations in $\widetilde{f}$ and $\widetilde{g}$ were relatively large, such perturbations were uncommon in our experiments and we still managed to obtain valid candidates. in the context of Problem 1.4. We justify adding noise uniformly since in practice we would expect this work to be applied on data which suffers from round off errors which tend to be uniformly distributed across the data.

\subsection{Bounded Coefficient Tests}

In this section we perform our tests on $f$ and $g$ whose coefficients are bounded and somewhat structured. The following steps detail the construction of our examples.

1. Generate $h_{1}, h_{2}, h_{3} \in \mathbb{R}[t]\left[\partial ;^{\prime}\right]$ where $\operatorname{deg}_{t} h_{i} \leq 2$ and $1 \leq \operatorname{deg}_{\partial} h_{i} \leq 2$.

2. Set $h_{i j}=h_{i j} /\left\|h_{i j}\right\|$ for $i=1,2,3$ and $1 \leq j \leq \operatorname{deg} h_{i}$. More precisely, $\left\|h_{i}\right\|=\operatorname{deg}_{t} h_{i}+1$. 
3. Compute $f=h_{1} \cdot h_{3}+\delta_{f}$ and $g=h_{2} \cdot h_{3}+\delta_{g}$, where the noise is distributed uniformly.

4. Run Algorithm 3, the Nearest With GCRD algorithm on $f$ and $g$.

5. If Nearest With $\operatorname{GCRD}(f, g)=1$ then ignore the result in the statistics.

The construction of these examples seems peculiar and counterintuitive, but it provides a suitable set of tests. The justification for the set of tests is to get an idea how the algorithm performs on random data that is not normalized, but is bounded with a random structure.

The table in Figure 1 details the relevant statistics obtained from running Algorithm 3. The table in Figure 2 provides the number of trivial GCRDs that occurred for a given $\rho$ and $\delta$.

Figure 1: Perturbation statistics for bounded coefficients

\begin{tabular}{|c|c|c|c|}
\hline$(\rho, \delta$, Reconstructed $)$ & Max & Average & Standard Deviation \\
\hline$(.5, .5, \widetilde{f})$ & 0.269443 & 0.022653 & 0.040056 \\
$(.5, .5, \widetilde{g})$ & 0.233732 & 0.025051 & 0.037960 \\
$\left(.5, .5, \widetilde{f}_{w}\right)$ & 0.269443 & 0.023507 & 0.038163 \\
$\left(.5, .5, \widetilde{g}_{w}\right)$ & 0.233732 & 0.023625 & 0.032776 \\
\hline$(.5, .1, \widetilde{f})$ & 0.047198 & 0.010184 & 0.011120 \\
$(.5, .1, \widetilde{g})$ & 0.061742 & 0.011490 & 0.013434 \\
$\left(.5, .1, \widetilde{f}_{w}\right)$ & 0.045457 & 0.009629 & 0.010101 \\
$\left(.5, .1, \widetilde{g}_{w}\right)$ & 0.049726 & 0.010082 & 0.010634 \\
\hline$(.5, .01, \widetilde{f})$ & 0.233401 & 0.006653 & 0.030810 \\
$(.5, .01, \widetilde{g})$ & 0.166854 & 0.005394 & 0.021614 \\
$\left(.5, .01, \widetilde{f}_{w}\right)$ & 0.233401 & 0.005647 & 0.027110 \\
$\left(.5, .01, \widetilde{g}_{w}\right)$ & 0.166854 & 0.005647 & 0.018869 \\
\hline$(.5, .001, \widetilde{f})$ & 0.283611 & 0.006625 & 0.037541 \\
$(.5, .001, \widetilde{g})$ & 0.182687 & 0.004582 & 0.025346 \\
$\left(.5, .001, \widetilde{f}_{w}\right)$ & 0.283611 & 0.006277 & 0.037003 \\
$\left(.5, .001, \widetilde{g}_{w}\right)$ & 0.182687 & 0.004317 & 0.024933 \\
\hline
\end{tabular}


Figure 2: Trivial Numeric GCRDs for Bounded Coefficients

\begin{tabular}{|c|c|c|}
\hline$\rho$ & $\delta$ & Trivial GCRD \\
\hline .5 & .5 & 9 \\
.5 & .1 & 3 \\
.5 & .01 & 3 \\
.5 & .001 & 0 \\
\hline .05 & .5 & 95 \\
.05 & .1 & 42 \\
.05 & .01 & 0 \\
.05 & .001 & 2 \\
\hline
\end{tabular}

\subsection{Normalized Tests}

In this section we perform our tests on $f$ and $g$ that were normalized and added noise. The following steps detail the construction of our examples.

1. Generate $h_{1}, h_{2}, h_{3} \in \mathbb{R}[t]\left[\partial ;^{\prime}\right]$ where $\operatorname{deg}_{t} h_{i} \leq 2$ and $1 \leq \operatorname{deg}_{\partial} h_{i} \leq 2$.

2. Compute $f=\frac{h_{1} \cdot h_{3}}{\left\|h_{1} \cdot h_{3}\right\|}+\delta_{f}$ and $g=\frac{h_{2} \cdot h_{3}}{\left\|h_{2} \cdot h_{3}\right\|}+\delta_{g}$, where the noise is distributed uniformly.

3. Run Algorithm 3, the Nearest With GCRD algorithm on $f$ and $g$.

4. If Nearest With $\operatorname{GCRD}(f, g)=1$ then ignore the result in the statistics.

The table in Figure 3 details the relevant statistics obtained from running Algorithm 3. The table in Figure 4 provides the number of trivial GCRDs that occurred for a given $\rho$ and $\delta$. 
Figure 3: Perturbation statistics for normalized $f$ and $g$

\begin{tabular}{|c|c|c|c|}
\hline$(\rho, \delta$, Reconstructed $)$ & Max & Average & Standard Deviation \\
\hline$(.5, .5, \widetilde{f})$ & 0.108768 & 0.015880 & 0.022180 \\
$(.5, .5, \widetilde{g})$ & 0.158056 & 0.016359 & 0.024371 \\
$\left(.5, .5, \widetilde{f}_{w}\right)$ & 0.052060 & 0.010584 & 0.010404 \\
$\left(.5, .5, \widetilde{g}_{w}\right)$ & 0.097296 & 0.011577 & 0.014039 \\
\hline$(.5, .1, \widetilde{f})$ & 0.058105 & 0.017043 & 0.014834 \\
$(.5, .1, \widetilde{g})$ & 0.063637 & 0.025312 & 0.017561 \\
$\left(.5, .1, \widetilde{f}_{w}\right)$ & 0.057251 & 0.012524 & 0.013178 \\
$\left(.5, .1, \widetilde{g}_{w}\right)$ & 0.046134 & 0.019487 & 0.012614 \\
\hline$(.5, .01, \widetilde{f})$ & 0.053014 & 0.005987 & 0.009114 \\
$(.5, .01, \widetilde{g})$ & 0.057982 & 0.006851 & 0.009757 \\
$\left(.5, .01, \widetilde{f}_{w}\right)$ & 0.031045 & 0.004263 & 0.006710 \\
$\left(.5, .01, \widetilde{g}_{w}\right)$ & 0.038133 & 0.005996 & 0.007523 \\
\hline$(.5, .001, \widetilde{f})$ & 0.066271 & 0.003778 & 0.009018 \\
$(.5, .001, \widetilde{g})$ & 0.093175 & 0.004416 & 0.011236 \\
$\left(.5, .001, \widetilde{f}_{w}\right)$ & 0.067082 & 0.003556 & 0.009915 \\
$\left(.5, .001, \widetilde{g}_{w}\right)$ & 0.038098 & 0.003213 & 0.006429 \\
\hline
\end{tabular}

Figure 4: Trivial Numeric GCRDs for Normalized $f$ and $g$

\begin{tabular}{|c|c|c|}
\hline$\rho$ & $\delta$ & Trivial GCRD \\
\hline .5 & .5 & 6 \\
.5 & .1 & 0 \\
.5 & .01 & 2 \\
.5 & .001 & 1 \\
\hline .05 & .5 & 86 \\
.05 & .1 & 22 \\
.05 & .01 & 2 \\
.05 & .001 & 1 \\
\hline
\end{tabular}




\section{Conclusions and Future Work}

We have developed a framework for approximate differential polynomials and demonstrated an algorithm for computing the greatest common right divisor of two approximate differential polynomials. This corresponds to finding a representation of the common solutions of two approximate linear differential operators.

Our algorithm makes use of the (unstructured) SVD approach introduced in Corless et al. (1995), and works well when differential polynomials with a non-trivial GCRD are nearby. Unfortunately, it also suffers some of the same drawbacks, especially when the nearest polynomials with a non-trivial GCRD are relatively far away. Even more so, the lack of a geometric root space (as for conventional polynomials) makes the analysis substantially more difficult. One possible remedy we are exploring is to this is to use a structured matrix approach, as explored in Beckermann and Labahn (1998), Botting et al. (2005), Kaltofen et al. (2006). In particular, Riemannian SVD-like methods would seem relatively easy to adapt in this case (though again, analysis will not be easy).

Another problem is the factorial-like scaling introduced by multiple differentiations when constructing the (inflated) differential Sylvester matrix. This leads to numerical instability when the degree in $\partial$ gets even modestly large. Some row scaling may alleviate this somewhat, but an alternative differential resultant formulation would seem a better approach overall, and is a path we are investigating.

The algorithms described in this paper have been implemented in Maple and are primarily based on the LinearAlgebra package. This allows flexibility in determining the method used to solve the linear systems and dealing with other problems such as content removal and numerical stability.

In general the approximate GCRD algorithm performs quite well for low degree GCRDs and low noise. If the degree of the GCRD increases relative to the degrees of $f$ and $g$ then $\widehat{V}+\Delta \widehat{V}$ becomes more unstructured and the perturbations become larger which leads to unsuitable numeric GCRDs. In the case of degree one or two GCRDs we were still able to reconstruct meaningful answers even if the noise was outside of our search radius because the matrix still retained a Sylvester-like structure.

Despite the speculative nature of our algorithm we find that it works reasonably well on the test data when we have a priori bound on the noise and choose a search radius accordingly. Approximating GCRDs of higher order 
becomes more difficult with the unstructured approach as the perturbations in the data will become larger. If other assumptions are made about the structure of $f$ and $g$ and the distribution of noise then it would be possible to obtain a better approximate GCRD by exploiting this underlying structure.

\section{References}

B. Beckermann and G. Labahn. When are two numerical polynomials relatively prime? Journal of Symbolic Computation, 26:677-689, 1998.

D. Bini and V. Pan. Polynomial division and its computational complexity. Journal of Complexity, 2(3):179-203, 1986.

B. Botting, M. Giesbrecht, and J.P. May. Using the Riemannian SVD for problems in approximate algebra. In Proc. Workshop on Symbolic-Numeric Computation (SNC'05), pages 209-219, 2005.

M. Bronstein and M. Petkovšek. On Ore rings, linear operators and factorisation. Programmirovanie, 20:27-45, 1994.

R. M. Corless, P. M. Gianni, B. M. Trager, and S. M. Watt. The singular value decomposition for polynomial systems. In Proc. International Symposium on Symbolic and Algebraic Computation (ISSAC'95), pages 189-205, 1995.

R. M. Corless, S. M. Watt, and L. Zhi. QR factoring to compute the GCD of univariate approximate polynomials. IEEE Transactions on Signal Processing, 52(12), 2004.

J. von zur Gathen, J. Gerhard. Modern Computer Algebra. Cambridge University Press, New York, NY, USA, 2 edition, 2003.

M. Giesbrecht and M. Kim. Computing the Hermite form of a matrix of Ore polynomials. Journal of Algebra, 376:341-362, 2013.

G. Golub and C. van Loan. Matrix Computations. Johns Hopkins University Press, Baltimore, USA, 4th edition, 2013.

E. Kaltofen, Z. Yang, and L. Zhi. Structured low rank approximation of a Sylvester matrix. In Proc. Workshop on Symbolic-Numeric Computation (SNC'05), pages 69-83, 2005. 
E. Kaltofen, Z. Yang, and L. Zhi. Approximate greatest common divisors of several polynomials with linearly constrained coefficients and singular polynomials. In Proc. International Symposium on Symbolic and Algebraic Computation (ISSAC'06), pages 169-176, 2006.

N. Karmarkar and Y. N. Lakshman. Approximate polynomial greatest common divisors and nearest singular polynomials. In Proc. International Symposium on Symbolic and Algebraic Computation (ISSAC'96), pages 35-39, 1996.

Z. Li and I. Nemes. A modular algorithm for computing greatest common right divisors of Ore polynomials. In Proc. International Symposium on Symbolic and Algebraic Computation (ISSAC'97), pages 282-289, 1997.

O. Ore. Theory of non-commutative polynomials. Annals of Mathematics. Second Series, 34:480-508, 1933.

T. Sasaki and M. Sasaki. Polynomial remainder sequence and approximate GCD. ACM SIGSAM Bulletin, 31:4-10, 1997.

Z. Zeng and B. H. Dayton. The approximate GCD of inexact polynomials. In Proc. International Symposium on Symbolic and Algebraic Computation (ISSAC'04), pages 320-327, 2004. 